\title{
Multifunctional cocoon silk prepared by plasma treatment
}

\begin{abstract}
Multifunctionality has become the key metrics for cocoon silk applications as textile materials. In this study, plasma treatment, a simple and effective method, is used to prepare multifunctional cocoon silks with electrical conductivity and water repellency. The results show $\mathrm{Cu} / \mathrm{CuO}$ particles are covered on the surface of cocoon silks, which ion's attachment amounts are not affected by different varieties of cocoon silks. Comparing with resistance values, the conductivity of $\mathrm{Cu}$-sputtered FS was $\sigma=1 \times 10^{-}$ ${ }^{6} \mathrm{Scm}^{-1}$, however, the conductivity of Cu-sputtered DS was $\sigma=5 \times 10^{-10} \mathrm{Scm}^{-1}$. The capillary height of pure DS cocoon silk is $2.5 \mathrm{~mm}$ within $9 \mathrm{~h}$, obviously higher than water-repellent treatment samples. Nevertheless, the strain of ion-sputtered cocoon silk is slightly decreasing. This multifunctionalzed cocoon silk satisfies the market demand for natural "smart" products, and is a promising practical material for use in the textile industry.
\end{abstract}

Keywords: cocoon silk, plasma treatment, conductivity, water repellency
Volume 3 Issue 5 - 2017

\author{
Xiaoru Li, Jinfa Ming, Jing Yang, Dagang Miao, \\ Xin Ning \\ Industrial Research Institute of Nonwovens and Technical \\ Textiles, Qingdao University, China
}

Correspondence: Jinfa Ming, Industrial Research Institute of Nonwovens and Technical Textiles, College of Textiles \& Clothing, Qingdao University, Qingdao, 26607I, China, Tel 8 65328595 3358, Fax 8653285953076 ,

Email mingjinfa@qdu.edu.cn, jinfa.ming@gmail.com

Received: November 27, 2017 | Published: December 22, 2017
Abbreviations: FS, fresh cocoon silk; DS, dry cocoon silk; SF, silk fibroin; EDS, energy dispersive x-ray spectroscopy

\section{Introduction}

Cocoon silks are composed of two silk proteins: sericin and fibroin. Fibroin is a semi crystalline polymer of natural fibrous protein mainly consisting of highly $\beta$-sheet crystals and non-crystalline part (micro voids and amorphous structures). Sericin is amorphous acting as an adhesive binder to maintain the fine filaments of fibroin core and the overall structural integrity of cocoon. ${ }^{1}$ Silk fibers are one of most important materials extensively used in textile industry. Due to different degumming process in silk processing from cocoons to the finished products, cocoon silks are divided into different types, such as fresh cocoon silk (FS) and dry cocoon silk (DS). Since degumming imposes a relative harsh environment on cocoon silk, the possibility of changes occurring in silk microstructure and mechanical properties etc. have been considered. ${ }^{2}$

Due to unique properties, cocoon silks can be applied in many fields such as textile and biomaterial etc. For the study of textile materials, more conditions are studied on the effect of tensile properties of degummed silk. The extrinsic (the location from which silk is collected within the cocoon, and the color of silk) and therefore controllable factors (storage condition prior to testing, and different styles of reeling) can affect the properties more than intrinsic ones. ${ }^{3}$ The complexity of silk proteome is analyzed and identified 500 proteins from seven silks. The results show fibroin and sericin are the most abundant proteins in all seven silk. At the same time, except for fibroin and sericin, protease inhibitors, enzymes and proteins of unknown function are more abundant than other proteins. ${ }^{4} \beta$-sheet crystal is determined the stiffness, strength, and optical properties of silk, which structural changes is occurred in the stretching of silk fibers. ${ }^{5}$ Moreover, other types of cocoon silk (African wild cocoon silk) are also studied and revealed that cocoon shells are multilayered and porous structures constructed from highly cross-linked fibers. ${ }^{6}$ However, as biomedical materials, cocoon silk is dissolved in different solution systems (i.e. lithium bromide, calcium chloride, formic acid/calcium chloride, ${ }^{7}$ formic acid/hydroxyapatite, ${ }^{8}$ ionic liquid, ${ }^{9}$ etc.) to regenerate single/multi level silk fibroin (SF) materials. ${ }^{10}$ In preparing SF materials, controlling the self-assembly of SF molecules is crucial, which is determined the physical and chemical properties of regenerated materials. ${ }^{11}$

Here, we introduce plasma treatment to prepare multifunctional cocoon silks at room temperature. $\mathrm{Cu} / \mathrm{CuO}$ particles are sputtered on the surface of cocoon silks, and then hexamethyldisiloxane is coated to protect conductive layer, obtaining cocoon silks with electrical conductivity and water repellency. Many characterizations are used to analysis the properties of cocoon silks.

\section{Materials and methods}

\section{Materials}

Cocoon silks containing DS and FS are bought from Shandong province, China. The specification of silk was 20/22 dtex. All other chemical reagents are bought from Sinopharm Chemical Reagent Co. Ltd. All tests were performed under constant temperature and humidity condition $\left(20^{\circ} \mathrm{C}\right.$ and $\left.65 \% \mathrm{RH}\right)$.

\section{Sample preparation}

Plasma treatment is performed using magnetron sputtering machine (Beijing Technol. Co., LTD, China). The treatment system has one large chamber. The nitrogen gas is fed into this chamber to ensure the ion sputtering environment at low pressure. $\mathrm{Cu}$ particles (thickness: $130 \mathrm{~nm}$ ) are sputtered on the surface of DS and FS cocoon silks under a 6 A current by magnetron sputtering machine at room temperature. The target used in this study is commercial available $\mathrm{Cu}$ $\left(99.99 \%\right.$ purity). Deposition parameters are base pressure $\left(5 \times 10^{-4} \mathrm{~Pa}\right)$, working pressure $(0.2 \mathrm{~Pa})$, air flow rate $(25 \mathrm{sccm})$, target-substrate distance $(10 \mathrm{~cm})$, and sputtering speed $\left(30 \mathrm{~cm} \mathrm{~min}^{-1}\right)$. In addition, $\mathrm{CuO}$ particles are also sputtered on the surface of DS and FS cocoon silks under oxygen environment at low pressure. 


\section{Water-repellent treatment of sputtered cocoon fibers}

In order to protect $\mathrm{Cu} / \mathrm{CuO}$ particles conductive layer and obtain water repellency property, water-repellent monomer hexamethyldisiloxane is used. Water-repellent treatment is prepared by Plasma treat 400 (Plasma treat, Germany). Then, multifunctional fibers with electrical conductivity and water repellency are obtained under atmospheric conditions.

\section{Characterization}

Morphological test: The surface morphology of DS/FS cocoons silks containing sputtered $\mathrm{Cu} / \mathrm{CuO}$ and water-repellent treatment fibers are scanned under scanning electron microscopy (Phenom ProX, PHENOMWORLD, and Netherlands). Samples for SEM experiment are observed with gold coating. At the same time, energy dispersive X-ray spectroscopy (EDS) is employed to determine the elemental composition.

Conductivity test: Before testing, sputtered $\mathrm{Cu} / \mathrm{CuO}$ samples are standing for $24 \mathrm{~h}$ at standard condition $\left(20^{\circ} \mathrm{C}, 65 \% \mathrm{RH}\right)$. Then, the resistance values of samples are measured with a ZC-90G high insulation resistance meter (Shanghai Taiou Electric Co., Ltd.). According to equation (1), the electrical conductivity is calculated as follows:

$$
\sigma=L / R \times S
$$

Where $\sigma$ is the electrical conductivity of the fiber $\left(\mathrm{S} \mathrm{cm}^{-1}\right)$

$\mathrm{L}$ is the length of the cocoon measured $(\mathrm{cm})$

$\mathrm{R}$ is the resistance of the cocoon $(\Omega)$

$\mathrm{s}$ is the cross-sectional area of the fiber bundle $\left(\mathrm{cm}^{2}\right)$.

Water repellency test: To measure the water repellency of plasma coating, capillary effect test is used. During test process, it is observed the climbing distance of water on the water-repellent treatment cocoon silks in the set time.

Mechanical property: Cocoon silks containing sputtered $\mathrm{Cu} / \mathrm{CuO}$ and water-repellent treatment are measured using an automatic tensile tester (model 3365 electronic strength tester, Instron, Boston, USA). These samples are kept for $24 \mathrm{~h}$ at standard atmospheric conditions $\left(20^{\circ} \mathrm{C}, 65 \% \mathrm{RH}\right)$. During test process, distances between grips and test speeds are set to $20 \mathrm{~mm}$ and $10 \mathrm{~mm} \mathrm{~min}^{-1}$, and the pre-tension is $0.2 \mathrm{cN}$. An average of twenty measurements is reported as the mean \pm standard deviation for each sample.

\section{Statistical analysis}

All values are expressed as mean \pm standard deviation. Statistical differences are determined by a Mann-Whitney $U$ test (Independent $t$ test, SPSS).

\section{Results and discussion}

Cocoon silks, containing fibroin and sericin account for about $75 \mathrm{wt} \%$ and $25 \mathrm{wt} \%$, are one of the most important materials used in textile industry. ${ }^{1}$ At present, due to the difference reeling methods, there are two kinds of cocoon silks in the market, namely: DS and FS. The difference properties of these cocoon silks are studied by our team. ${ }^{2}$ In this research, we use DS and FS as raw materials to prepare multifunctional cocoon silks for textile industry application, which own electrical property and water repellency.

\section{Morphology of sputtered cocoon silk}

Figure 1 depicts the morphology of sputtered $\mathrm{Cu}$ particles on DS and FS cocoon silks. The surface of DS and FS cocoon silks is not smooth, existing a certain amount of sericin (Figure 2a) (Figure 2c). After sputtering, $\mathrm{Cu}$ particles are uniformly covered on the surface of cocoon silks (Figure 2b), which are confirmed by EDS (Figure 3a). At the same time, different varieties of cocoon silks are not affected $\mathrm{Cu}$ ion's attachment amounts. Moreover, Figure 2 also shows the morphology of sputtered $\mathrm{CuO}$ particles on DS and FS cocoon silks. These cocoon silks are smooth after sputtered $\mathrm{CuO}$ particles (Figure $2 \mathrm{~d}$ ). These $\mathrm{CuO}$ particles are also confirmed by EDS (Figure $3 \mathrm{~b}$ ).
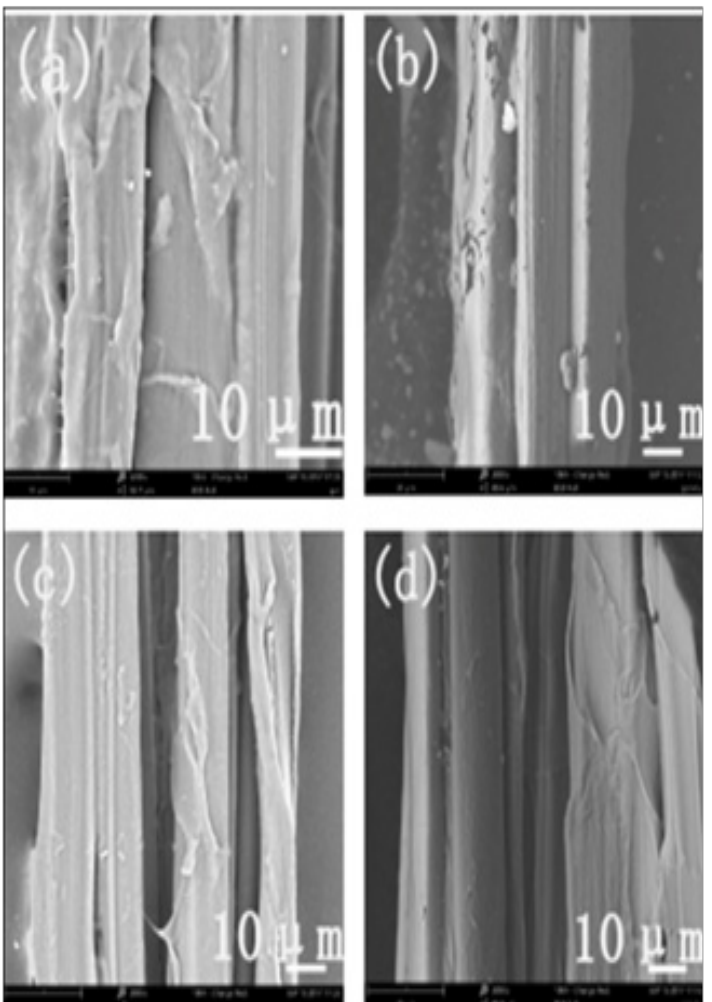

Figure I Morphological of sputtered $\mathrm{Cu}$ particles on DS and FS cocoon silks: (a) DS cocoon silk; (b) Cu-sputtered DS silk; (c) FS cocoon silk; (d) Cusputtered FS silk.

\section{Electrical properties of ion-sputtered cocoon silks}

The sputter coating significantly changes the surface characteristics of cocoon silk fibers. SEM observations clearly reveal the change in surface morphology of fibers sputter coated with $\mathrm{Cu}$ particles (Figure 1). For $\mathrm{Cu}$-sputtered cocoon silk, conductivity is found to be dependent on the dispersion of $\mathrm{Cu}$ particles. Through the resistance testing of samples, the conductivity of $\mathrm{Cu}$-sputtered FS was $\sigma=1 \times 10^{-}$ ${ }^{6} \mathrm{Scm}^{-1}$, however, the conductivity of $\mathrm{Cu}$-sputtered DS was $\sigma=5 \times 10^{-10}$ $\mathrm{Scm}^{-1}$. Thus, it shows the conductivity of $\mathrm{Cu}$-sputtered DS was better than FS. In addition, the conductivity of $\mathrm{CuO}$ is less than $\mathrm{Cu} . \mathrm{Cu}$ particles are used to prepare ion-sputtered conductive materials for further research. Therefore, in order to obtain excellent conductivity, $\mathrm{Cu}$ particles are prevented to be oxidized.

\section{Water repellency of ion-sputtered cocoon silks}

$\mathrm{Cu}$-sputtered DS cocoon silks are treated using water-repellent monomer hexamethyldisiloxane. After treatment, the morphology 
of $\mathrm{Cu}$-sputtered DS cocoon silk is shown in Figure 4a. In order to evaluate the water-repellent effect of samples, capillary effect is used. From Figure 5, Cu-sputtered DS cocoon silk with waterrepellent treatment has scarcely capillary effect within $1 \mathrm{~h}$. However, the capillary height of pure DS cocoon silk is achieved to $0.75 \mathrm{~mm}$, exhibiting excellent hydrophilic property. This is related to sericin containing a lot of hydrophilic groups. When test time is increased to $9 \mathrm{~h}$, the capillary height of pure DS cocoon silk is $2.5 \mathrm{~mm}$, higher than treated samples. It shows $\mathrm{Cu}$-sputtered DS cocoon silks with water-repellent treatment have a good water-repellent effect. In addition, water-repellent treatment samples are washed in deionized water under ultrasonic treatment for $30 \mathrm{~min}$. $\mathrm{Cu}$ particles layer is also covered on the surface of DS cocoon silks (Figure 4c). Water-repellent layer plays a protective role to prevent the falling of conductive layer. From literature, $\mathrm{Cu}$ (II) is involved in the natural spinning process of a silkworm helping to convert the concentrated silk fibroin solution into tough insoluble threads. ${ }^{12}$ At the same time, silk fibroin shows a low $\mathrm{Cu}$ ions absorption capacity of $10.8 \mathrm{mg} / \mathrm{g}$. The amino acid composition of silk fibroin mainly includes glycine, alanine and serine, which hardly plays a role of binding site for positive metal ions due to its weak polarity. However, physical adsorptions play a dominant role in $\mathrm{Cu}$ ions adsorption on the surface of silk fibroin..$^{13}$ In our work, the physical adsorptions are dominated in sputter- $\mathrm{Cu}$ ions on the surface of cocoon silks. For further work, in order to improve the binding fastness of sputtered-Cu ions, chemical bonding is essential.
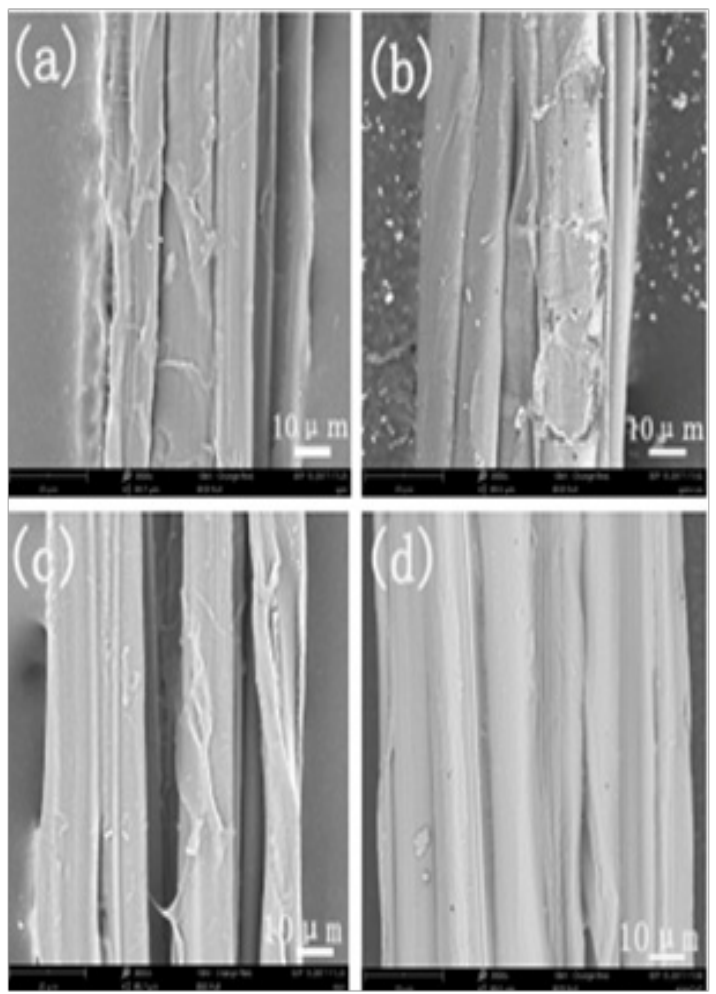

Figure 2 Morphological of sputtered $\mathrm{CuO}$ particles on DS and FS cocoon silks: (a) DS cocoon silk (b) CuO-sputtered DS silk (c) FS cocoon silk (d) CuO-sputtered FS silk.

\section{Mechanical properties of ion-sputtered cocoon silks}

The results of mechanical tests are shown in Figure $6 \&$ Table 1. The stress and strain are $0.73 \pm 0.15 \mathrm{MPa}, 22.50 \pm 2.43 \%$ and $1.47 \pm 0.25$ $\mathrm{MPa}, 20.91 \pm 3.71 \%$, respectively, for DS and FS cocoon silks. It exhibited the breaking strength of FS slightly higher than DS. When cocoon silks are ion-sputtered, the stress of samples was decreasing. The breaking strength of $\mathrm{CuO}$-sputtered samples is higher than $\mathrm{Cu}$ sputtered samples.

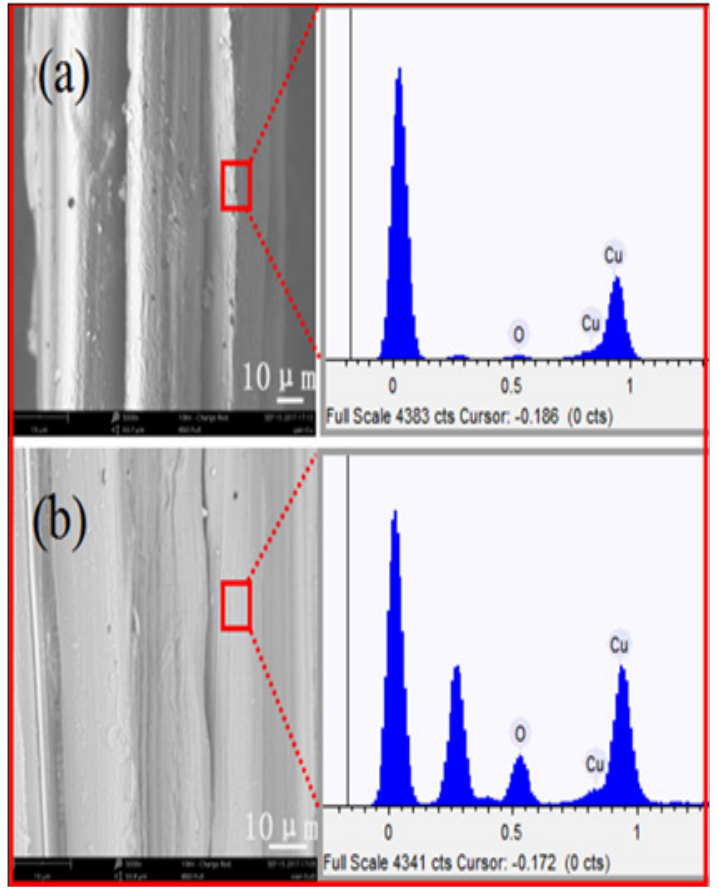

Figure 3 EDS of sputtered $\mathrm{Cu} / \mathrm{CuO}$ cocoon silks: (a) Cu particles, (b) $\mathrm{CuO}$ particles.
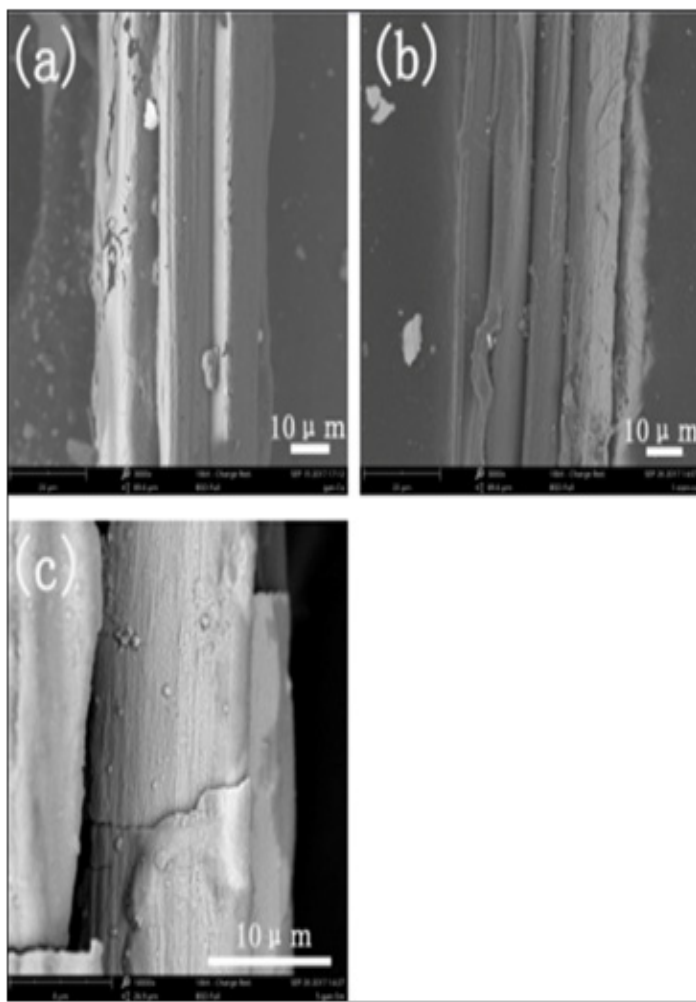

Figure 4 Morphology of Cu-sputtered DS cocoon silks treated by waterrepellent monomer. At the same time, samples are washed for different times: (a) $0 \mathrm{~min}$, (b) $5 \mathrm{~min}$, and (c) $30 \mathrm{~min}$. 

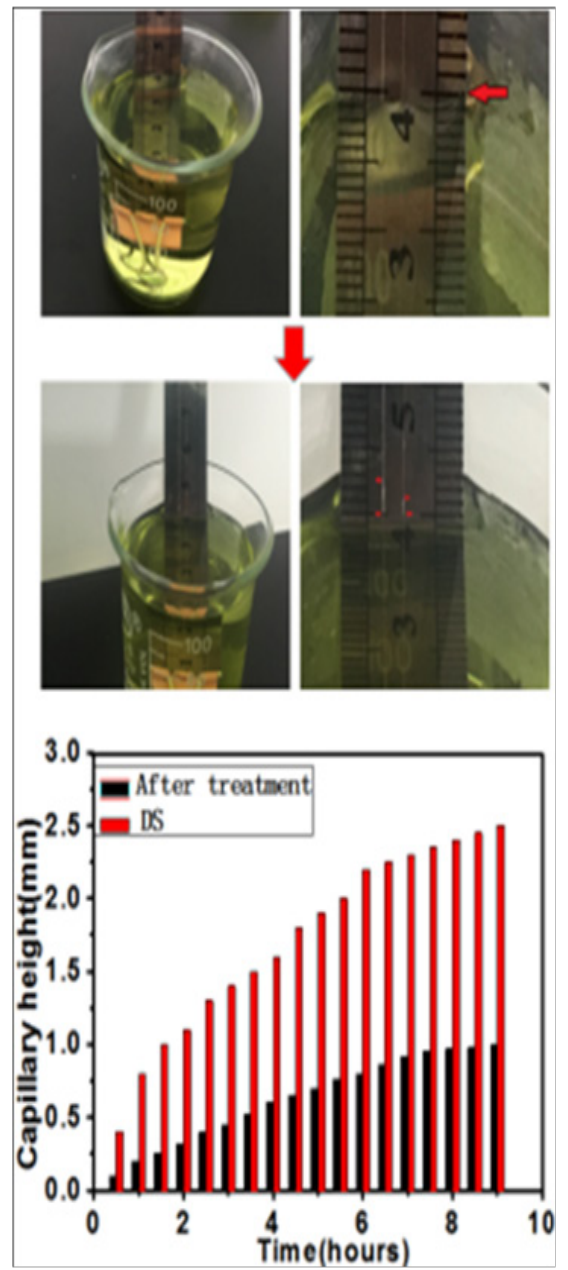

Figure $\mathbf{5}$ The water hydrophobic evaluation of Cu-sputtered DS cocoon silk treated by water-repellent monomer.

Table I Mechanical property of different cocoon silks prepared by ion sputtering method

\begin{tabular}{llll}
\hline Samples & Diameter $\left(\times 10^{-3} \mathbf{m m}\right)$ & Strain (\%) & $\begin{array}{l}\text { Stress } \\
(\mathbf{M P a})\end{array}$ \\
\hline DS & $1.0 \pm 0.25$ & $22.50 \pm 2.43$ & $0.73 \pm 0.15$ \\
FS & $0.86 \pm 0.45$ & $20.91 \pm 3.71$ & $1.47 \pm 0.25$ \\
DS-Cu & $1.12 \pm 0.32$ & $12.00 \pm 2.18$ & $0.57 \pm 0.16$ \\
FS-Cu & $1.08 \pm 0.25$ & $19.47 \pm 2.15$ & $0.86 \pm 0.23$ \\
& & & \\
DS-CuO & $1.28 \pm 0.32$ & $27.16 \pm 3.67$ & $0.58 \pm 0.17$ \\
FS-CuO & $0.96 \pm 0.28$ & & $1.07 \pm 0.22$ \\
& & $10.92 \pm 2.56$ &
\end{tabular}

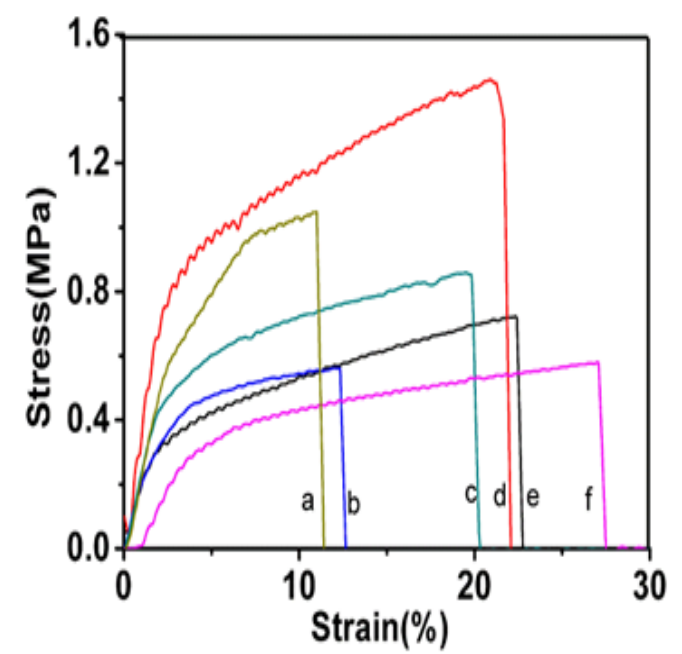

Figure 6 Mechanical properties of different cocoon silks prepared by ion sputtering method: (a) FS-CuO, (b) DS-Cu, (c) FS-Cu, (d) FS, (e) DS, and (f) DS-CuO, respectively.

\section{Conclusion}

Cocoon silks are one of most important materials extensively used in textile industry. In order to prepare multifunctional cocoon silks, plasma treatment is used. $\mathrm{Cu} / \mathrm{CuO}$ particles are covered on the surface of cocoon silks. The results show different varieties of cocoon silks are not affected ion's attachment amounts. Comparing with resistance values, the conductivity of $\mathrm{Cu}$-sputtered FS was $\sigma=1 \times 10^{-6} \mathrm{Scm}^{-1}$, however, the conductivity of Cu-sputtered DS was $\sigma=5 \times 10^{-10} \mathrm{Scm}^{-1}$. After water-repellent treatment, $\mathrm{Cu}$-sputtered DS cocoon silks have a good water-repellent effect. Moreover, when cocoon silks are ionsputtered to obtain more functionality, the stress of cocoon silk is decreasing slightly. Therefore, plasma treatment is a simple, effective method to prepare multifunctional cocoon silks, which is enlarged its application from textile materials to conductive materials etc. These multifunctional cocoon silks are used to prepare antistatic cocoon silk fabrics and "smart" fabric and so on.

\section{Acknowledgements}

This work was financially supported by National Natural Science Foundation of Shandong Province (BS2015CL017), China Postdoctoral Science Foundation (2016M592141), Shandong Postdoctoral Innovation Project (201603067), Qingdao Innovation Project (17-1-1-68-jch), the Open Project Program of Key Laboratory of Eco-textiles, Ministry of Education, Jiangnan University (No. KLET1511), the Cooperation Project of Zhongyuan University of Technology (20163702011482), and Industrial Research Institute of Nonwovens \& Technical Textiles, Qingdao University.

\section{Conflict of interest}

a. This manuscript is original work, and has not been copyrighted or published previously.

b. No other financial support or incentive has been provided for this manuscript. (All financial support is listed in this paper.)

c. I am one author signing on behalf of all co-authors of this manuscript. 


\section{References}

1. Jiang $\mathrm{P}$, Liu HF, Wang $\mathrm{CH}$, et al. Tensile behavior and morphology of differently degummed silkworm cocoon silk fibres. Mater Lett. 2006;60(7):919-925.

2. Ming JF, Pan QT, Pan FK, et al. Identification of dry and fresh cocoon silk. J Tex Eng Fashion Technol. 2017;2(6):00080.

3. Reed EJ, Bianchini LL, Viney C. Sample selection preparation methods and the apparent tensile properties of silkworm cocoon silk. Biopolymers. 2012;97(6):397-407.

4. Dong ZM, Zhao P, Wang C, et al. Comparative proteomics reveal diverse functions and dynamic changes of bombyx mori silk proteins spun from different development stages. J Proteome Res. 2013;12(11):5213-5222.

5. Numata K, Masunaga H, Hikima T, et al. Use of extension deformation based crystallisation of silk fibres to differentiate their functions in nature. Soft Matter. 2015;11(31):6335-6342.

6. Teshome A, Vollrath F, Raina SK, et al. Study on the microstructure of African wild silk cocoon shells and fibers. Int J Biol Macromol. 2012;50(1):63-68.
7. Yue XX, Zhang F, Wu HY, et al. A novel route to prepare dry-spun fibers from CacCl2-formic acid solution. Mater Lett. 2014;128(8):175-178.

8. Ming JF, Liu Z, Bie SY, et al. Novel silk fibroin films prepared by formic acid hydroxyapatite dissolution method. Mater Sci Eng C. 2014;37:48-53.

9. DM Phillips, LF Drummy, DG Conrady, et al. Dissolution and regeneration of Bombyx mori silk fibroin using ionic liquids. J Am Chem Soc. 2004;126(44):14350-14351.

10. Qi Y, Wang H, Wei K, et al. A review of structure construction of silk fibroin biomaterials from single structures to multi level structures. Int $J$ Mol Sci. 2017;18(3):237.

11. Dubey P, Murab S, Karmakar S, et al. Modulation of self-assembly process of fibroin an insight for regulating the conformation of silk biomaterials. Biomacromolecules. 2015;16(12):3936-3944.

12. Zhou L, Chem X, Dai WL, et al. X-ray photoelectron spectroscopic and raman analysis of silk fibroin $\mathrm{Cu}(\mathrm{II})$ films. Biopolymers. 2010;82(2):144-151.

13. Zhou WT, Hang HT, Du S, et al. Removal of copper ions from aqueous solution by adsorption onto novel polyelectrolyte film coated nanofibrous silk fibroin nonwovens. Appl Surf Sci. 2015;345:169-174. 